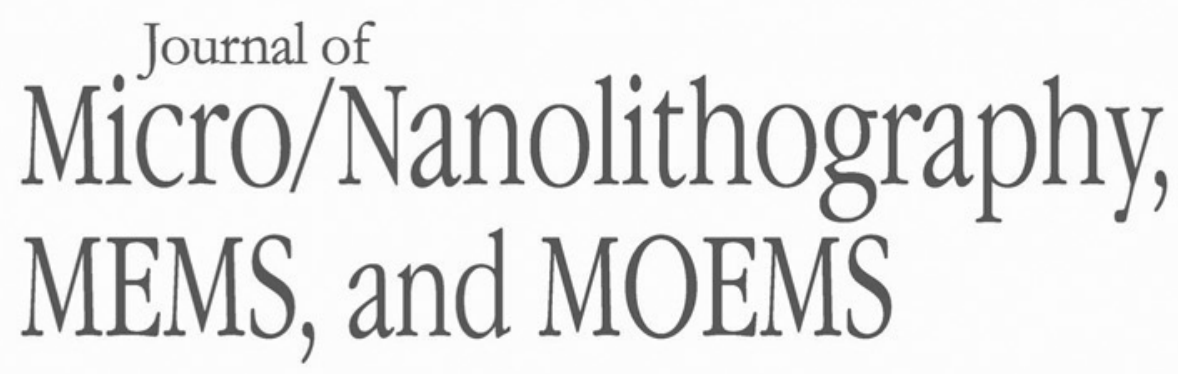

Nanolithography.SPIEDigitalLibrary.org

\title{
Effective-CD: a contribution toward the consideration of line edge roughness in the scatterometric critical dimension metrology
}

Bartosz Bilski

Karsten Frenner

Wolfgang Osten 


\title{
Effective-CD: a contribution toward the consideration of line edge roughness in the scatterometric critical dimension metrology
}

\author{
Bartosz Bilski, Karsten Frenner, ${ }^{*}$ and Wolfgang Osten \\ Institut für Technische Optik, Universität Stuttgart, Pfaffenwaldring 9, 70569 Stuttgart, Germany
}

\begin{abstract}
We present an overview of our research on the relation between line edge roughness (LER) and optical critical dimension metrology (OCD). Referring to a known fact that LER does have an impact on OCD, we discuss a novel approach that allows for its better understanding. Namely, we show that, in the presence of LER, one can observe a characteristic scatterometry-measured CD offset, which we call effective-CD. The fact that the effective-CD is characteristic renders it to be a good means of accessing the information about LER present on the CD. To assure the completeness of this overview, we begin by reviewing some previously published results, which have drawn our attention and first led us to observing the characteristic influence of LER on a CD measurement. Next, we extend our model-based simulations to confirm the presence of the effectiveCD for complex-roughness models, and finally, we demonstrate an experimental verification of our effective-CD hypothesis. We are convinced that our approach will help to better understand the impact of LER on a CD measurement and will be considered a useful contribution to the development of measurement methods for challenging scenarios, in which realistic CD is affected by the presence of LER. ๑ The Authors. Published by SPIE under a Creative Commons Attribution 3.0 Unported License. Distribution or reproduction of this work in whole or in part requires full attribution of the original publication, including its DOI. [DOI: 10.1117/1.JMM.16.2.024002]
\end{abstract}

Keywords: semiconductor industry; Moore's law; critical dimension metrology; critical dimension scanning electron microscopy; line edge roughness; scatterometry; model-based simulations.

Paper 16101 received Jun. 23, 2016; accepted for publication Feb. 21, 2017; published online May 17, 2017.

\section{Introduction}

For decades, the semiconductor industry continues to drive patterning solutions by reducing device dimensions or, more specifically, by printing nodes of a continuously shrinking critical dimension (CD). As $\mathrm{CD}$ values shrink further, the phenomenon of line edge roughness (LER) gains substantial attention. ${ }^{1}$ LER is defined as "a deviation of a feature edge (as viewed top-down, $e$ ) from a smooth, ideal shape $(d)$," or more specifically as ${ }^{1}$

$\operatorname{LER}=3 \cdot \sigma=3 \cdot \operatorname{RMS}(e-d)$.

The "gains substantial attention" is expressed by statements like "LER can become the most significant source of linewidth control problems for features $\leq 50 \mathrm{~nm}$."1 In the light of such statements, it is clear why optical scientists are striving to provide process engineers with a solution that would contribute to solving the stated linewidth control problem.

\section{Line Edge Roughness Indeed has an Impact on a CD Measurement}

The question whether or not LER has an impact on the optical ( = scatterometric) CD measurement has received attention from at least a few groups. There are different specific approaches to the challenge, such as selection of

*Address all correspondence to: Karsten Frenner, E-mail: frenner@ito. uni-stuttgart.de regular or Mueller matrix spectroscopic ellipsometry, Fourier scatterometry, or a combination of diffraction and scattering modeling. ${ }^{2-5}$ Despite the differences, all these approaches have two things in common. They are all optical methods, and they all deliver a positive answer: LER indeed does have an impact on an optical CD measurement. This way, essentially, our demonstration in this section is not novel in itself, but it serves as a good starting point to presenting our treatment of the subject. Additionally, what to our knowledge is a new insight, it serves as an illustration of the necessity to use a realistic roughness model to achieve realistic results of LER simulation (as opposed to a simplified square or sinusoidal "roughness" used in other works).

We start our investigation by computing a scatter signature of a perfect photoresist line grating ( = LER is exactly zero) of $C D=50 \mathrm{~nm}$. We utilize this $C D$ value throughout the rest of our simulations to represent a typical CD printed by a modern lithographic process. For the purpose of this demonstration, we fix the incidence angle at $\alpha=78 \mathrm{deg}$, while the conical angle remains zero [ = plane of incidence is perpendicular to the grating lines, see Fig. 3(a) in a later section of this paper] and vary the incident wavelength in an approximately visible range. Next, following the footsteps of our predecessors, ${ }^{2,3}$ we disturb the ideal lines of the grating by applying a sinusoidal roughness to their edges (in a way that the mean $C D$ is still exactly $50 \mathrm{~nm}$ ) and compute the corresponding scatter signature. Having done so, we compare the two scatter signatures by plotting the difference between them (see Fig. 1). As you can clearly see, the difference is nonzero. The only conclusion that can follow is that 


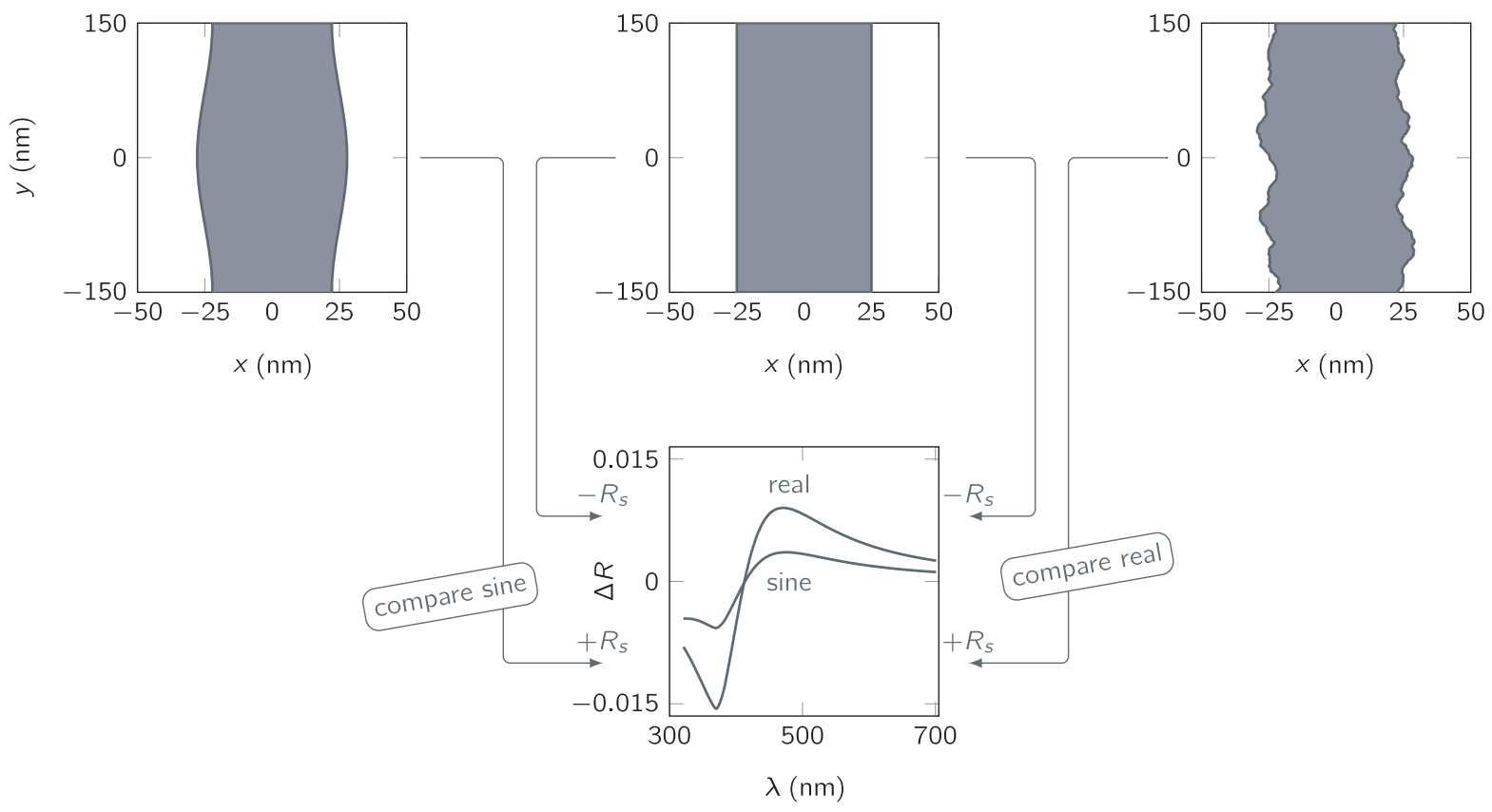

Fig. 1 LER indeed has an impact on a scatterometric CD measurement, but its magnitude is different, depending on whether we consider a realistic roughness model or a simplified, sinusoidal "roughness."

LER indeed has an impact on a scatterometric CD measurement.

In a further step, we apply a realistic roughness model ( $=$ its spectrum resembles the spectrum of a low-pass filter, see Fig. 2$)^{1}$ to the edges of the grating, always ensuring that the mean $\mathrm{CD}$ is still exactly $50 \mathrm{~nm}$; compute the corresponding scatter signature and compare it, as before, with the scatter signature of an ideal grating, see again Fig. 1. Two observations can be made as follows:

- the original observation that LER indeed has an impact on a scatterometric CD measurement remains valid and

- the magnitude of the stated impact is different when compared with the case of sinusoidal "roughness." Given the fact that our investigation is simulationbased ( $=$ no error sources present) with very strict ( = sub-one-per-mill) convergence criteria, we consider it not to be a simulation "artifact," but a real effect.

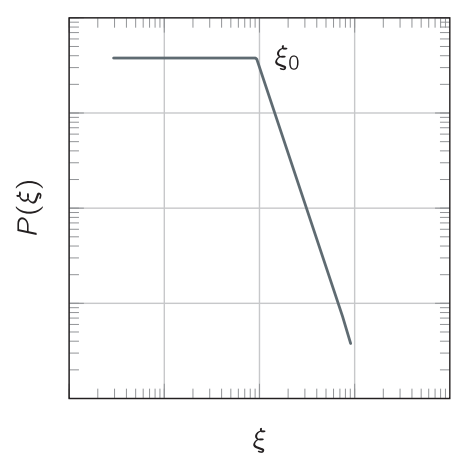

Fig. 2 A schematic representation of a realistic power spectrum of LER. To generate multiple and different stochastic rough profiles, we use a random number generator and assign a random phase to each spatial frequency $\xi$. In this paper, the cut-off frequency $\xi_{0}$ is such that $1 / \xi_{0}=100 \mathrm{~nm}$. The log-log slope is $m=-3$, which is usually a good value. ${ }^{6}$
For the latter reason, in all our subsequent considerations we will be using only a realistic roughness, as it is only reasonable for us to assume that the results generated by a realistic roughness model would be more realistic, too.

\section{Characteristic Pattern in the Impact that the Line Edge Roughness Exerts on the Scatter Signatures}

In this section, we continue comparing the computed scatter signatures of rough and ideal photoresist line gratings. In the interest of the completeness (and standaloneness) of this paper, we believe it is necessary to demonstrate what our approach is since the first steps. This results, as far as the first part of this section is concerned, in some overlap with our previous publication. ${ }^{7}$

Initially, we use the setup we present in Fig. 3(a), which, according to the location of the plane of incidence, is called 0 -deg azimuthal angle setup. As you can see, we take the $s$-polarized incidence into consideration, which is why in a certain coordinate system we can speak of a 0 -deg polarization. Unless otherwise indicated, we use the presented grating geometries throughout the remainder of this paper.

For the first comparison, we vary such geometric parameters like incidence angle $\alpha$, grating height $h$, or incident wavelength $\lambda$, and compute the corresponding reflected $s$-polarized scatter signatures of rough gratings. Given that in our case $\lambda / p>1$, "scatter signatures" specifically means "zeroth diffraction order," as this is the only one that is propagating to the far field for all incidence angles $\alpha$. We generate rough gratings by applying a realistic roughness model with a few increments of a $\sigma$-value of roughness (see Fig. 3), whereas its other parameters-its correlation length (equivalent to $\xi_{0}$, see Fig. 2) and roughness exponent (equivalent to the slope in Fig. 2)-always remain constant. The computing method that we use in the process is our in-house implementation of the rigorous coupled wave analysis (RCWA) method. ${ }^{8}$ We model the roughness 
(a)

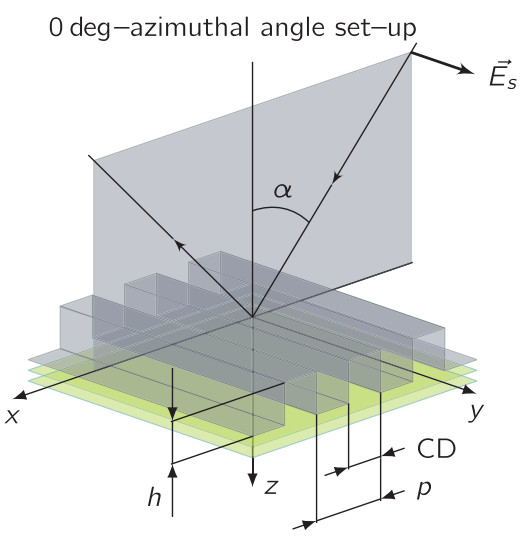

(b)

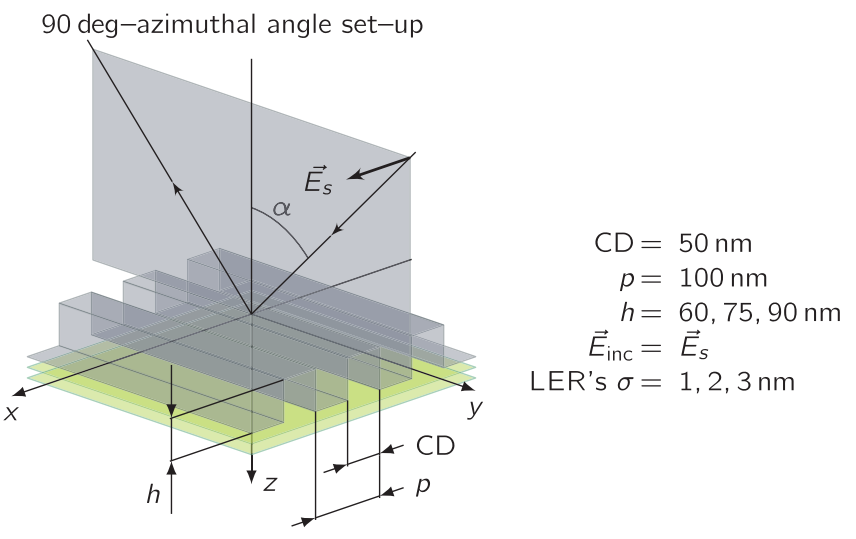

Fig. 3 The two scatterometric setups that we use for our simulations. (a) 0-deg azimuthal angle setup and (b) 90-deg azimuthal angle setup.

as constant in $z$ direction ( $=$ because we use only one layer of the staircase approximation), whereas in $x$ and $y$ directions, we expand it into $55(-27 \ldots+27)$ Fourier modes. In the context of convergence and in the context of what we present below, this precision provides sufficient quality of the computed results. A similar statement applies to the selected length of the "unit cell" in $y$ direction: $300 \mathrm{~nm}$ is enough to sufficiently capture the variations of a rough edge with a typical power spectrum. Even though only one example of a "unit cell" with a rough line is shown in Fig. 1, we tried more than 20 of them. All of them were exhibiting the same effect that we describe just below, which makes it very plausible that we are in fact describing a real scatterometric effect and not just a stochastic artifact-that we, despite our best efforts, failed to understand in its full complexity.

The next step is critical to understanding the way we look at scatterometry data and the conclusions we draw from it. In addition to computing the signatures of rough gratings, we also compute a scatter signature of a perfect grating presented in Fig. 3. Most importantly, when doing so, we also allow the $\mathrm{CD}$ of this perfect grating to depart a little from its nominal value (but this applies only to the $\mathrm{CD}$; all other parameters, including pitch, remain fixed). This way, instead of computing just one scatter signature of a perfect grating, we compute a certain envelope of these.

The only operation that remains is to present the two families of scatter signatures in one plot, on top of each other.

In the big picture, see Fig. 4(a), all scatter signatures are indistinguishable from one another. It can naturally be explained by the fact that we apply little variation to the $\mathrm{CD}$, as well as by the fact that we apply little roughness. However, when we take a much closer look by peeking into two "zoom windows," we start noticing an interesting pattern, see Figs. 4(b) and 4(c). Namely, the scatter signatures of rough gratings seem to experience an offset with respect to the scatter signature of a perfect grating of $C D=50 \mathrm{~nm}$. Interestingly, the more the LER, the more the offset. Even more interestingly, the offsets seem to be regular, in a sense that each of the scatter signatures of rough gratings seems to closely follow one particular scatter signature from the envelope.

The above observation may just be a coincidence without any deeper meaning; it could be that the offsets seem so regular in just these two "zoom windows." In order to verify whether this is the case or not, we quantify the offsets by assigning, for each incidence angle, a CD value to them. An approximate assignment can be carried out simply by
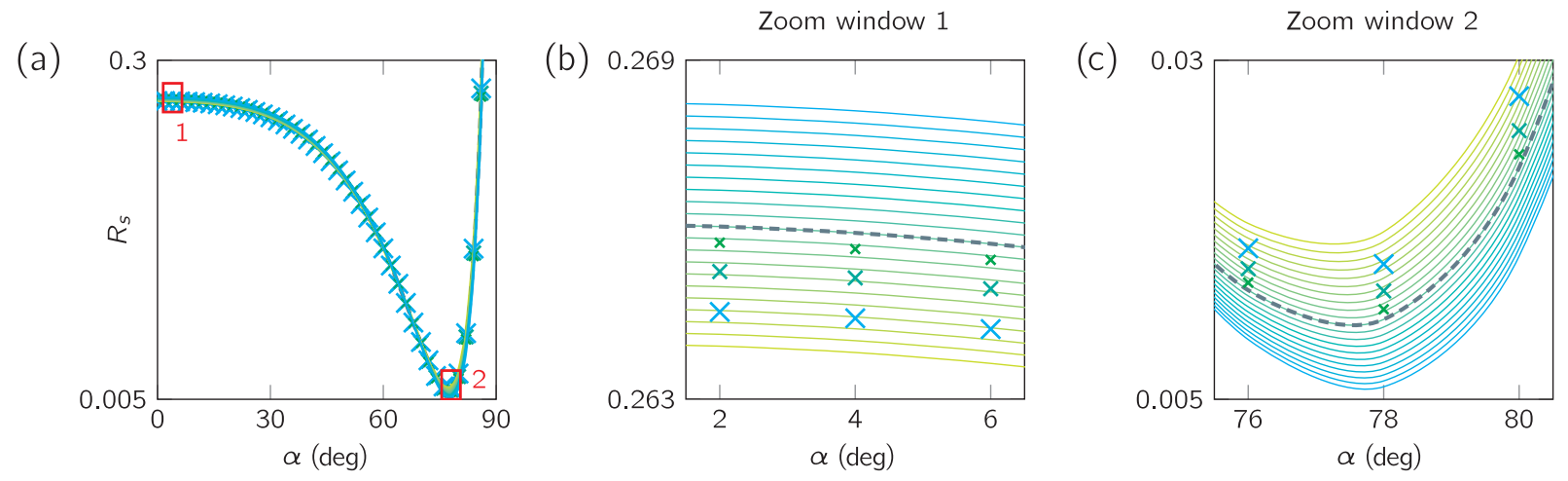

\begin{tabular}{|c|c|c|c|c|c|c|}
\hline$C D$ & 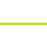 & $49 \mathrm{~nm}$ & $=-\ldots$ & $50 \mathrm{~nm}$ & 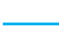 & $51 \mathrm{~nm}$ \\
\hline LER's $\sigma$ & $x$ & $1 \mathrm{~nm}$ & $x$ & $2 \mathrm{~nm}$ & $x$ & $3 \mathrm{~nm}$ \\
\hline
\end{tabular}

Fig. 4 When we look into "zoom windows," we notice that scatter signatures of rough gratings closely follow scatter signatures of ideal gratings of different $C D$. 


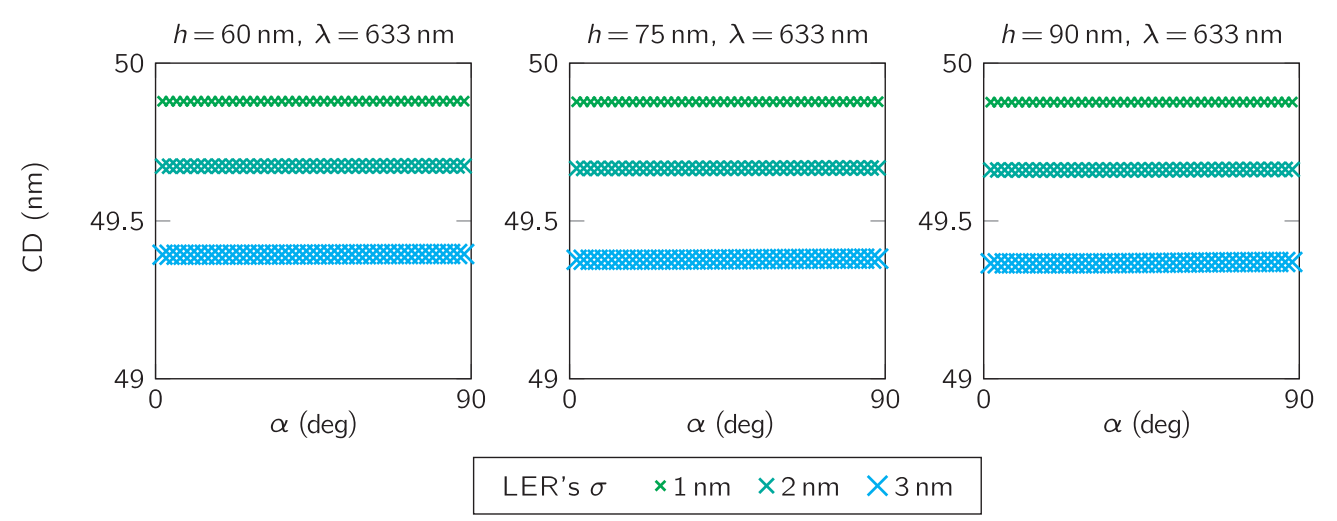

Fig. 5 The CD offsets as a function of incidence angle $\alpha$ in 0-deg azimuthal angle setup are very regular.

looking at "zoom windows" like Fig. 4(b). To assign precise numbers, we make use of the fact that the signatures within the envelope exhibit a fair degree of linearity and perform a spline interpolation per incidence angle. We show the results of this operation in Fig. 5. As you can see, the offsets indeed are regular for the whole spectrum of incidence angles $\alpha$, which in turn means that the initial observation in Fig. 4 would look the same in any other "zoom window." Additionally, the observation is practically identical for a couple of considered grating heights $h$.

However, the most important conclusion is a very disturbing one: from the scatterometric point of view, LER measurement seems to be impossible in 0-deg azimuthal angle setup, as scatter signatures of rough and perfect gratings overlap, rendering one indistinguishable from the other. As scatterometric measurement involves a step of model-based reconstruction, it is unable to "decide" on its own which of the two cases is actually being measured and thus is unable to deliver a true reconstruction.

Although it may seem like it, the fact that scatterometry seems to be unable to distinguish between rough and perfect gratings does not mean that scatterometry is intrinsically unfit for a LER metrology application. We demonstrate this by repeating the above exercise again, but this time in a 90-deg azimuthal angle setup, where "90 deg" represents the plane of incidence rotated by $90 \mathrm{deg}$ compared with the previous case, see Fig. 3(b). Consequently, we can also speak of 90-deg polarization in this case. In Fig. 6, we present the best-fitting CD values based on reflected $s$-polarized scatter signatures. As you can see, apart from the fact that the observed effect is still regular and observable at any incidence angle or grating height, there is a distinct and key difference-this time, instead of being indistinguishable from a narrower $\mathrm{CD}$, the exact same rough line is indistinguishable from a broader CD.

Up to this point, we have considered just one wavelength in our investigations. To verify whether or not our observations still hold when the wavelength becomes a floating parameter, we again repeat the above investigation for $\alpha=78 \mathrm{deg}$ (we have already determined that the effect is incidence angle invariant, so we keep that parameter fixed) in 0-deg and 90-deg azimuthal angle setups and vary the wavelength $\lambda$ in the range corresponding roughly to the visible spectrum of light. We present the results of this investigation in Fig. 7. You can see there that, as far as the considered wavelengths are concerned, the observed effect can also be regarded to be wavelength-invariant.

Even though the above results indicate that our observation about the $\mathrm{CD}$ offsets is quite general, there exists yet another concern about the usability of the results. Namely, is it sufficient to consider only one layer of RCWA's sidewall approximation? Among other things, such a simplification limits the realism of our simulations by implying that the roughness on the sidewall is anisotropic, and that the sidewall angle is exactly $90 \mathrm{deg}$, and that there are no roundings. Another concern might be the following-could it be that all of the above observations is a result of an "undocumented feature" ( = bug) of our RCWA implementation? To verify

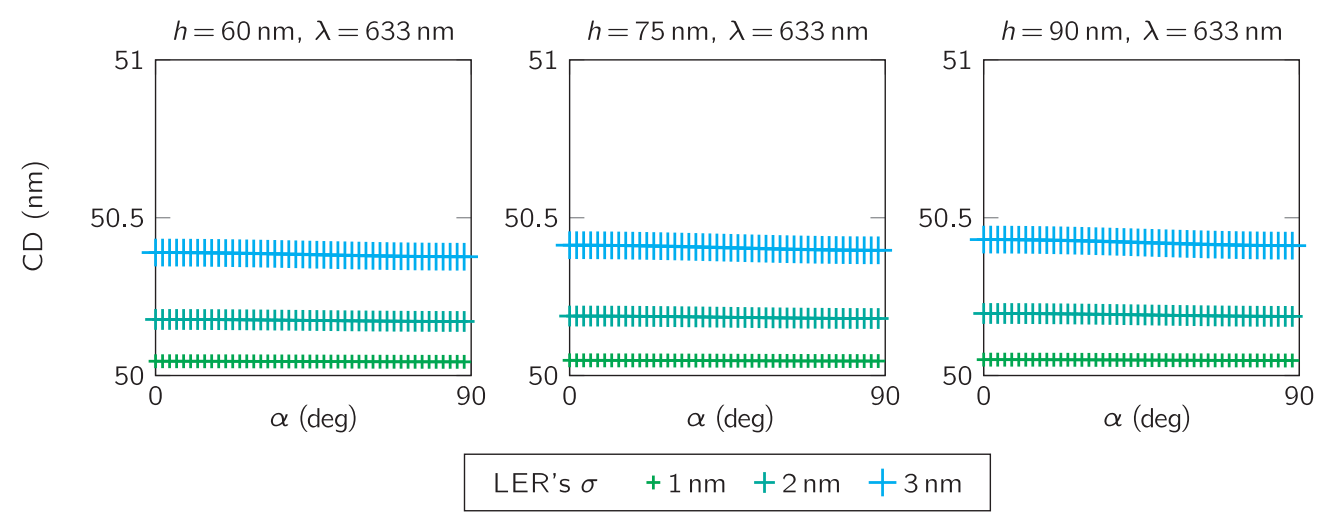

Fig. 6 The $C D$ offsets as a function of incidence angle $\alpha$ in 90-deg azimuthal angle setup are very regular. 

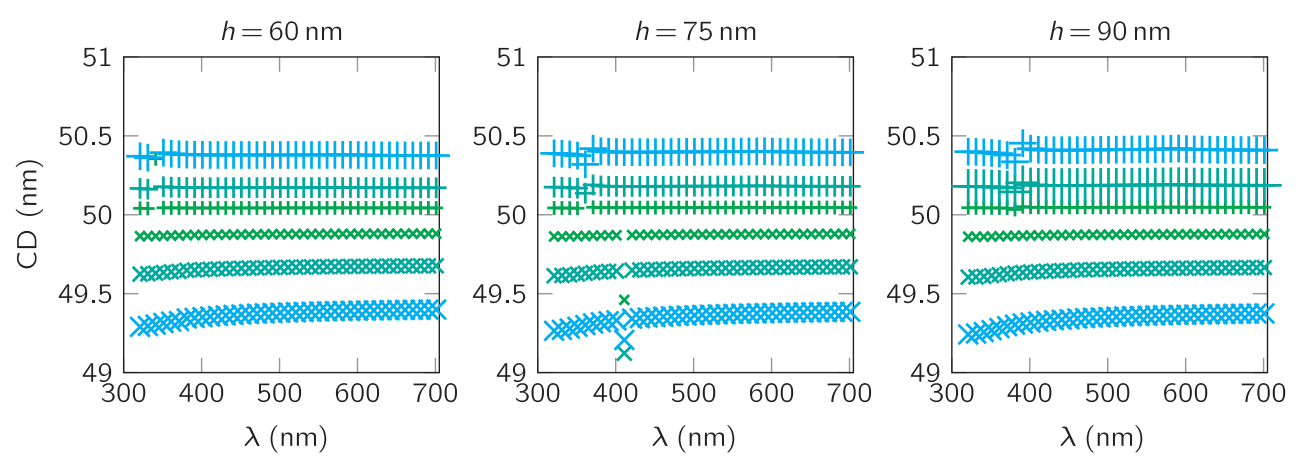

$$
\begin{array}{r}
0 \text { deg-azimuthal angle set-up: LER's } \sigma \times 1 \mathrm{~nm} \times 2 \mathrm{~nm} \times 3 \mathrm{~nm} \\
90 \mathrm{deg} \text {-azimuthal angle set-up: LER's } \sigma \quad+1 \mathrm{~nm}+2 \mathrm{~nm}+3 \mathrm{~nm}
\end{array}
$$

Fig. 7 The CD offsets as a function of incident wavelength $\lambda$ in both setups are very regular.
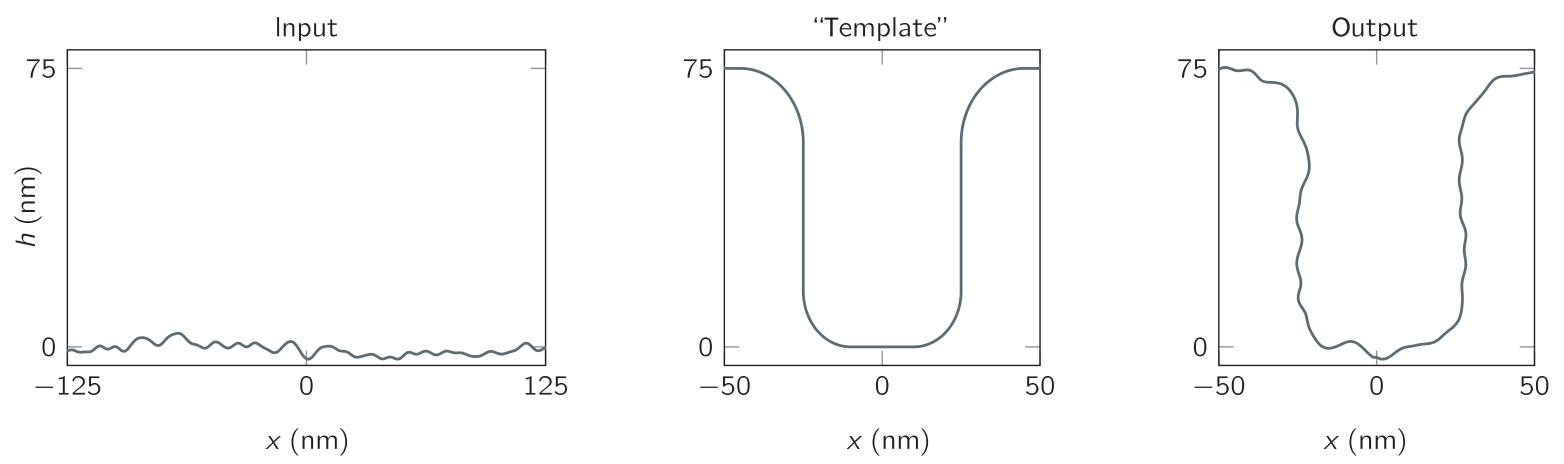

Fig. 8 We use a simple deform operation to transform a two-dimensional rough surface into a model of an isotropically rough line.

these concerns, we run a next set of simulations that utilize a more complex roughness model and another computing method.

We create a more complex rough line by deforming a two-dimensional, isotropically rough surface according to a

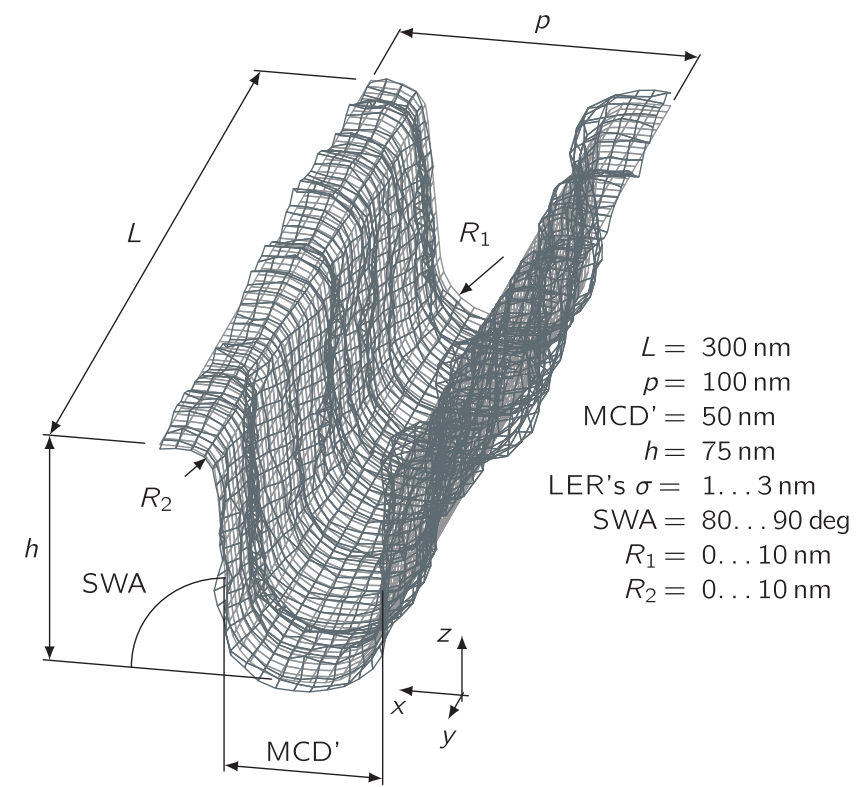

Fig. 9 An isotropically rough line that we use to cross-check whether or not our previous results are correct. "template," as schematically sketched in Fig. 8. The resulting rough profile is presented in Fig. 9. Because of the freedom with which we can shape the "template," the new rough profile does not exhibit the limitations we mentioned previously: the sidewall is now also $<90 \mathrm{deg}$, top and bottom roundings are present, and roughness on the sidewalls is isotropic. This last property poses a certain challenge to our LER investigation because of the way LER is typically defined: "deviation from a smooth ideal shape as viewed top-down." If in our case roughness varies also along the sidewall in $z$ direction, the question that is quite natural to ask is: what exactly is "roughness as viewed top-down" in such a case? It is important that we emphasize that this question is not a challenge to the simulations, but to the LER definition itself and to the metric in which results should be reported and interpreted. To utilize an easy metric, we report the subsequent results as a function of the $\sigma$-value of the rough surface that was used as an input to the deform-operation from Fig. 8. As such, $\sigma$-values are not 1:1 equivalent to the $\sigma$-values from the previous simulations (there, it was clear what a "topdown" roughness was); we also look at the new results when they still exist as scatter signatures (before best-fitting them to $\mathrm{CD}$ values).

On a simulation end, we use our in-house implementation of the differential method. ${ }^{9,10}$ Given that we already observed certain invariances, we no longer vary $\alpha$ nor $\lambda$ nor $h$. Instead, we introduce and vary nonzero roundings and sidewall angle $<90 \mathrm{deg}$ to verify whether the previous observations still hold. In $z$ direction, we consider 72 equidistant sections, 

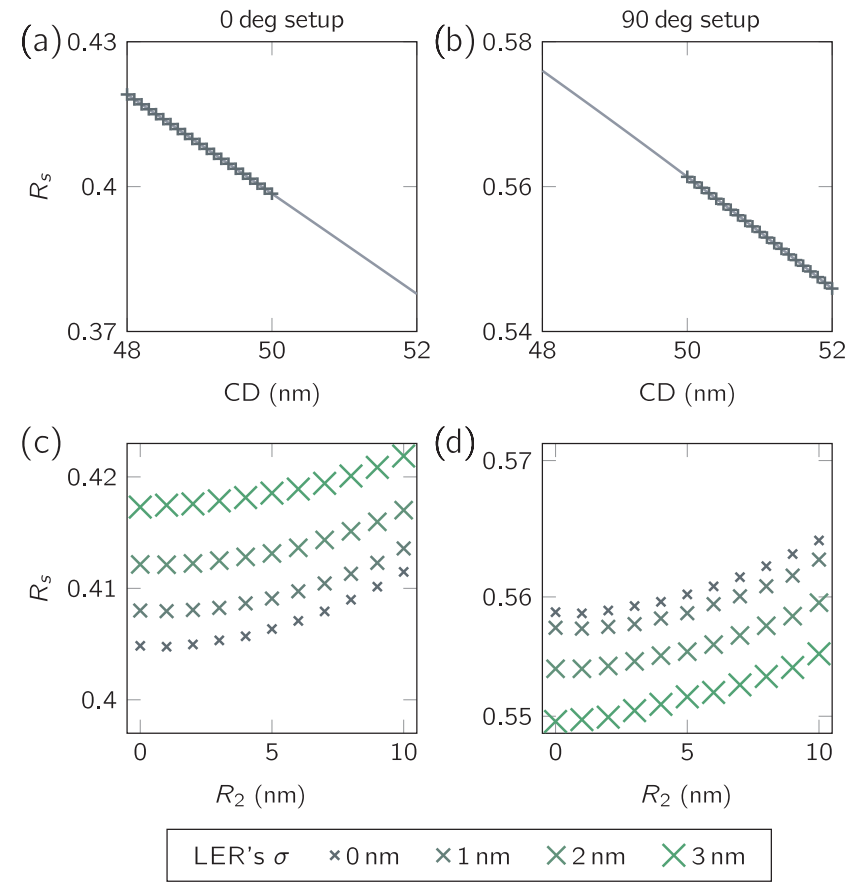

Fig. 10 Also in the case of realistically rough line, we see that its scatter signatures [(c), (d)] deliver expected CD offsets [(a), (b)]. You can find details in the text.

in which we determine the distribution of the refractive index, which, given the grating height $h$ of $75 \mathrm{~nm}$, results in a dense sampling of practically every $1 \mathrm{~nm}$. In $x$ and $y$ directions, we use $23(-11 \ldots+11)$ and $67(-33 \ldots+33)$ Fourier modes, respectively, which provides sufficient convergence of the results and an impressive (too impressive?) computing time of $24 \mathrm{~h}$ per datapoint.

The expectation is as follows: in 0-deg azimuthal angle setup, if the CD is supposed to shrink, the scatter signature should increase in value, see Fig. 10(a). In 90-deg azimuthal angle setup, on the other hand, if the $\mathrm{CD}$ is expected to expand, the scatter signature is expected to decrease in value [see Fig. 10(b)].

In Figs. 10(c) and 10(d), we see that the above expectations are indeed reflected in the simulation results. In 0-deg azimuthal angle setup, with increasing $\sigma$-values the scatter signature also increases in value, which is equivalent to a CD drop. In 90-deg azimuthal angle setup, with increasing $\sigma$-values the scatter signature decreases in value, which is equivalent to a $\mathrm{CD}$ expansion. The fact that data we obtained when using another (complex) roughness model and another simulation package confirm our initial observation is a good proof of the validity of these first observations (see Fig. 4) and the claims we make based on these.

For the sake of completeness of the picture, it is worth mentioning (and also is easy to deduce) that by making the complex roughness imitate our initial model ( = by setting roundings to zero and sidewall angle to $90 \mathrm{deg}$ ), we also get a confirmation of our initial observations. Results graphically look almost identical to results in Fig. 10, so for the lack of space and to avoid certain redundancy, we do not present them here.

Finally, we selected the 0-deg and 90-deg azimuthal angle setup for the clarity of the picture, but it is important to point out that our observations also hold at other azimuthal angles.

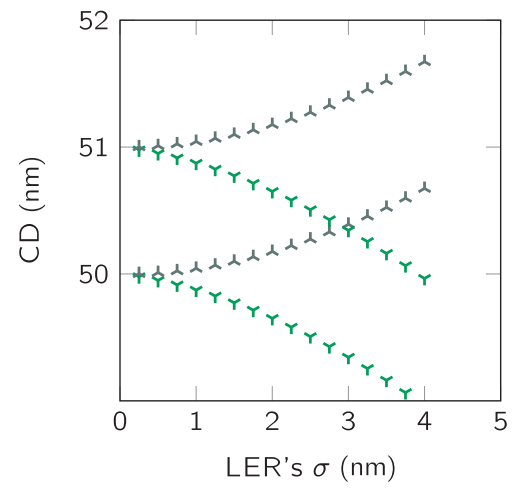

r 0 deg-azimuthal angle setup $\curlywedge 90 \mathrm{deg}$-azimuthal angle setup

Fig. 11 The CD offsets that we call the effective-CD. They are a polarization-dependent function of the $\sigma$-value of LER.

We demonstrate it briefly by looking at full pupils in Fourier scatterometric setup (details are presented in the reference introduced in Sec. 8).

\section{Concept of the Effective-CD}

Based on the observations we presented in the previous section, we come to the following conclusions:

- LER indeed has an impact on the optical CD measurement,

- the mentioned impact is irrespective of many parameters ( $\alpha, \lambda$, computing method, etc.), and

- depending on the setup considered, the more the LER is applied to the grating, the lower or the higher the effective-CD is observed. ${ }^{7,11}$

The term "effective-CD" represents the fact that any change of CD is purely virtual and is a result of a scatterometric reconstruction principle. In reality, in our simulations the "carrier CD" for the roughness is in all cases fixed at precisely $50 \mathrm{~nm}$. The fact that the exact same rough line, when considered at two setups (and, consequently, two perpendicular polarizations), delivers two distinct values of the effective-CD is a powerful means to detect and measure LER. You only need to perform a double CD measurement, at 0 -deg and 90-deg azimuthal angles, determine the effective-CD difference, and project it to the $\sigma$-value of the roughness, using a dependency similar to the one presented in Fig. 11.

In an extra comment, we want to mention that $\mathrm{CD}=50 \mathrm{~nm}$ is not a one and only $\mathrm{CD}$ value that generates these generalizable results. We draw similar conclusions in its vicinity; specifically, when repeating our investigation for another "carrier CD" of $51 \mathrm{~nm}$. There, we again see a pair of parabolas, which behave practically identical to the ones that we obtained for a CD of $50 \mathrm{~nm}$, see Fig. 11.

\section{Experimental Effective-CD Observation: The Approach}

To observe the effective-CD experimentally, we take a focus-exposure matrix (FEM) wafer, as this type of wafer is one of the most routinely used in calibrations and adjustments of a lithographic process. A typical FEM wafer is a two-dimensional matrix of targets, whereby each target is 


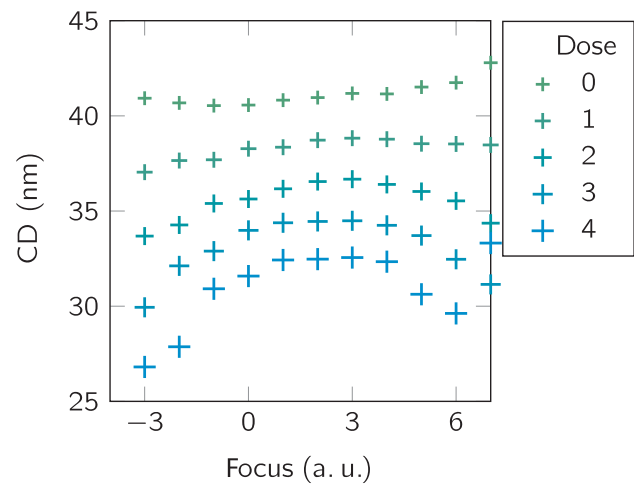

Fig. 12 Bossung curves obtained from a FEM. Dose and focus, as in the next figures, are in arbitrary units. By courtesy of ASML.

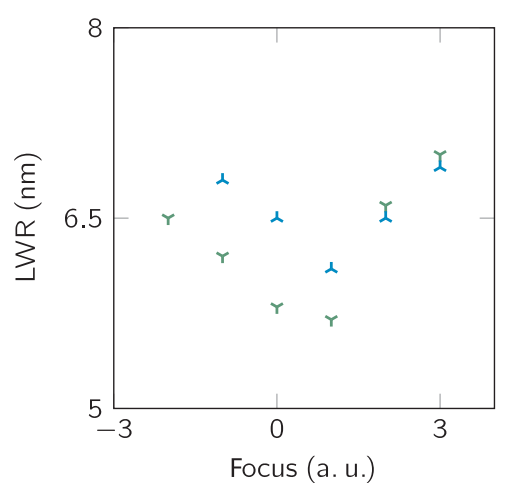

Fig. 13 A CD-SEM scan of a FEM wafer we use for the experiment shows that indeed various levels of LER are present. We present the data as originally reported by CD-SEM software ( $=$ as LWR), but, knowing that for uncorrelated roughness it is $L W R=\sqrt{2} L E R$, it is easy to get an idea what LER values are. By courtesy of ASML.
Nominal focus
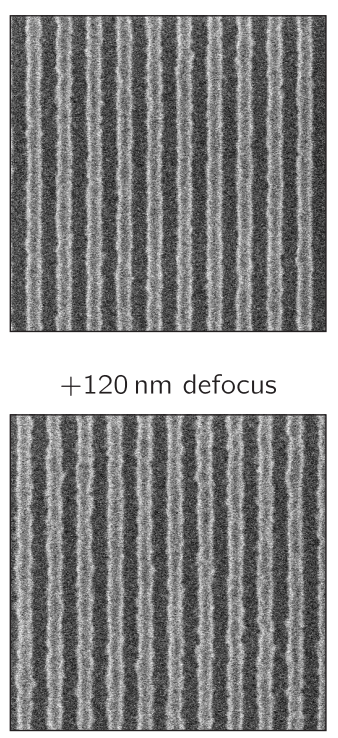

$+120 \mathrm{~nm}$ defocus
$+60 \mathrm{~nm}$ defocus

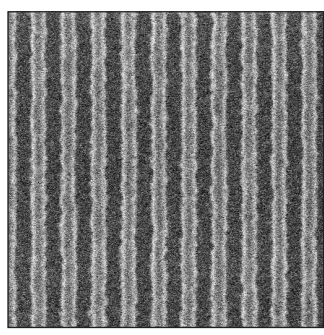

$+180 \mathrm{~nm}$ defocus

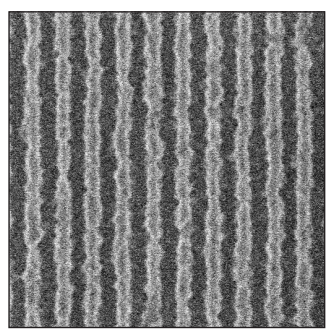

Fig. 14 CD-SEM scans illustrating various levels of LER on a FEM (not the one we used in the experiment). By courtesy of ASML. exposed with different parameters of focus and dose from within the process window and beyond. ${ }^{12}$ Typically, all the $\mathrm{CD}$ values that are printed by all dose $\times$ focus combinations are presented as so-called Bossung curves, see Fig. 12. These in turn help to determine the optimum dose $\times$ focus parameter range ("process window") that best prints the intended CD.

Also in our case FEM is an array of targets printed with different combinations of dose and focus values, where each target in fact contains two sub-targets: two line gratings, rotated by 90 deg with respect to each other. Using a certain wafer coordinate system, we name these sub-targets simply: 0-deg and 90-deg targets, see Fig. 15 later in this section. We present the example Bossung curves obtained from our FEM in Fig. 12. There, we see that there exists a dose value ( = FEM "column") that prints the CD of ca. $40 \mathrm{~nm}$ at pretty relaxed (de)focus settings.

We chose an FEM wafer because, from the point of view of what we want to verify, the most important property of the FEM - that in fact comes "naturally" as an "extra feature"is that even within the best "column" of a FEM, the values of LER are not constant. They are lowest in the vicinity of the optimum focus value and they increase with an increasing (de)focus, see Figs. 13 and 14. Others have also reported about this property of a FEM. ${ }^{6}$ For later considerations, it is important to notice that for our particular FEM, the lowest detected LER is located at a slightly positive (de) focus level.

If the results of our simulations are true, we expect to observe what we schematically present in Fig. 15. There, in an ideal-world scenario, we have access to an ideal FEM "column" that prints exact same CD (or, in fact, "carrier $\mathrm{CD}$," because similar to our simulation-based considerations from the previous sections, also here we are dealing with a line of a given perfect CD that is expected to additionally carry LER on its both edges) at all (de)focus values. The best focus is in the center of the FEM, which means that also lowest LER value is located there. Together with increasing defocus, LER also grows symmetrically toward the edges of the FEM. For 0-deg targets, this means the following:

- in the center of the ideal FEM "column," LER is the lowest, so that CD offsets from the "carrier CD" are the lowest,

- at the edges of the ideal FEM "column," LER is the highest, so that CD offsets from the "carrier CD" are the highest, and

- in the center and at the edges, for 0-deg azimuthal angle, the effective-CD seems to be lower than the "carrier CD," whereas for the 90-deg azimuthal angle it seems to be higher.

For 90-deg targets, the results are of course expected to be anti-symmetric. For both targets, however, the difference $|\Delta \mathrm{CD}|$ between the two effective-CD plots should be quasiparabolic, with the minimum located where LER is lowest.

\section{Experimental Effective-CD Observation: The Results}

In Fig. 16, we present the experimental results obtained from a typical FEM wafer using an experimental instrumentation 


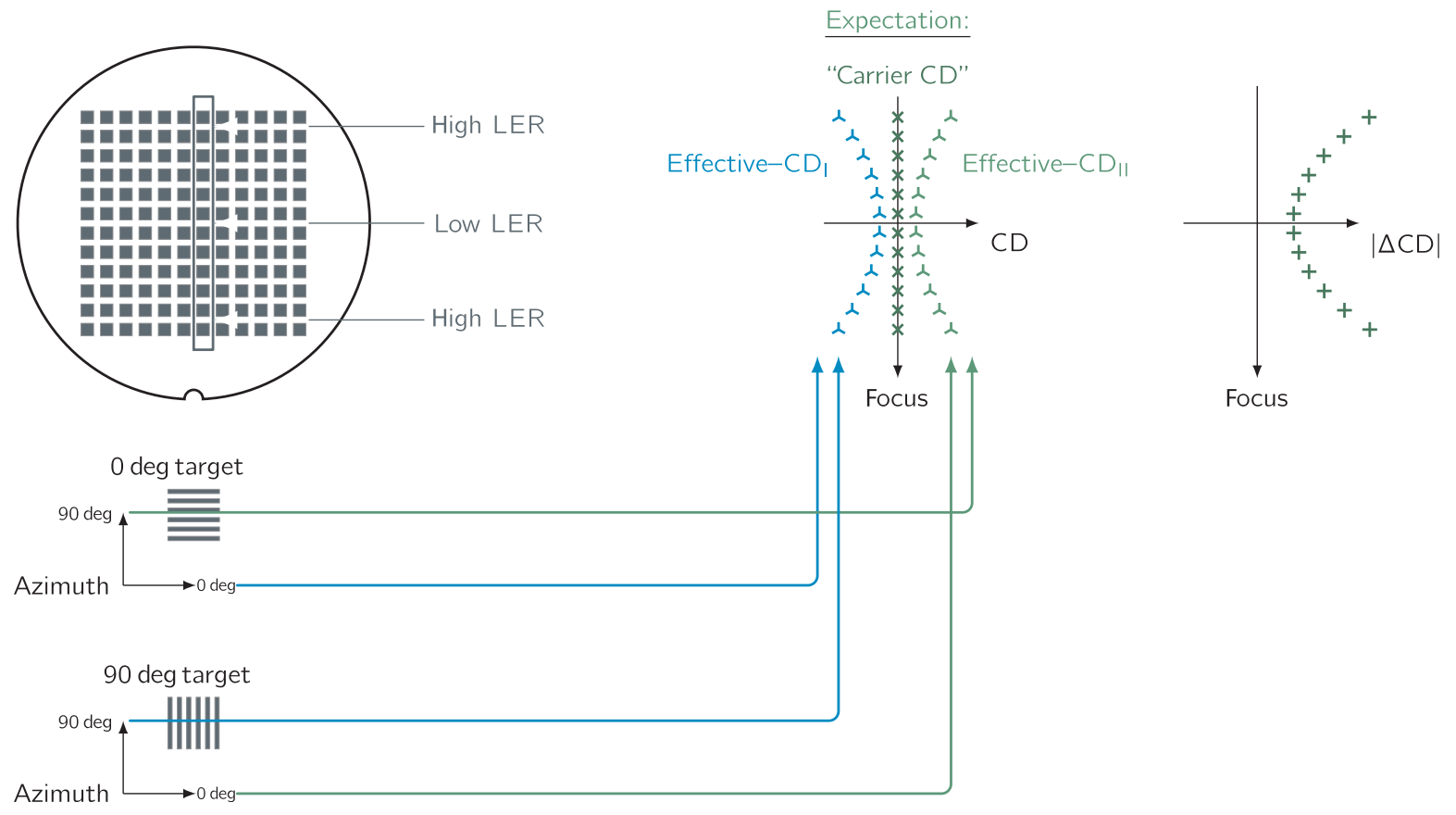

Fig. 15 An ideal FEM would contain a "column" with a constant "carrier CD" for all focus levels. Because of the fact that LER is not constant though, we expect different levels of effective-CD across that column. For the two setups and two targets, we naturally expect the results to be anti-symmetric, but they should always result in a quasiparabolic effective-CD difference $|\Delta C D|$.

that, among others, embodies the 0-deg and 90-deg azimuthal angle setups and is routinely used by our project partner ASML for the purpose of scatterometric CD metrology. Given the fact that we use a standard FEM, a standard dense-line pattern, a well-understood process, and metrology recipes, we benefit from the fact that the correlation between scatterometry- and CD-SEM-reported CD values in our case is very good, $>0.99$. This high correlation naturally applies to the "classical" scatterometric reconstruction scheme, that
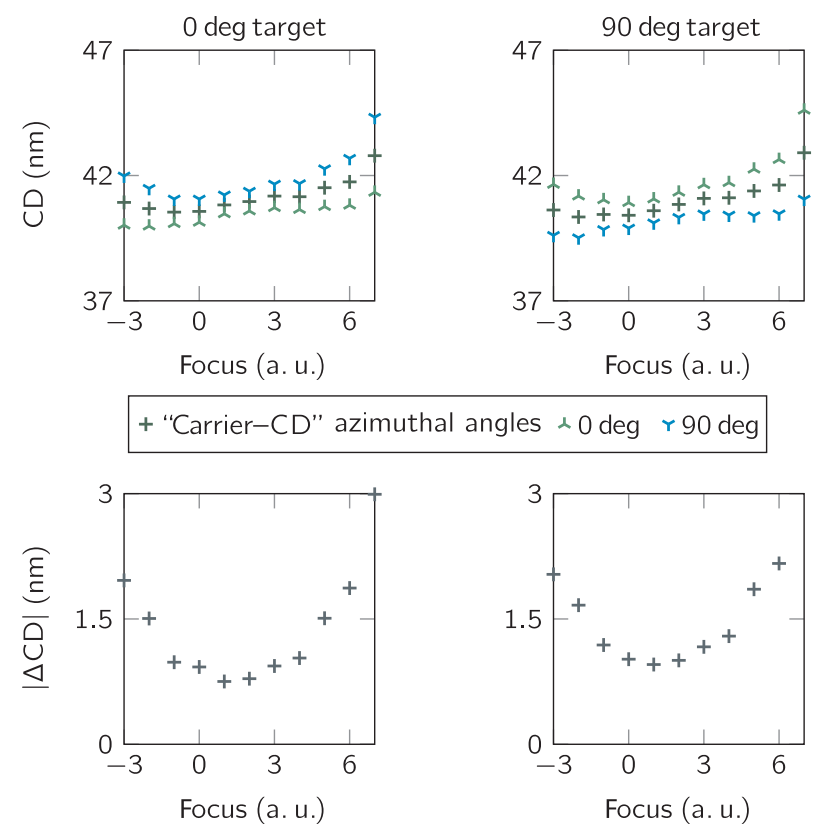

Fig. 16 The results of a scatterometric CD measurement on a real-life FEM wafer exhibit the presence of the effective-CD and confirm what is expected of it. is, the scheme that is not aware of the effective-CD concept. This is why in this case, even though the data labeled as "carrier CD" come from the experimental scatterometric instrumentation, we rely on them completely.

As we are dealing with a real-life FEM, we are not fortunate enough to work exactly at the isofocal point, meaning that at different focus levels a slightly different CD is printed. In fact, similar to our previous considerations, we should say "carrier CD is printed," because we know that not only lines are printed, but also lines with LER on their edges. It is quite natural to expect that by not being at the isofocal point we cannot reproduce the constant "carrier CD" at all focus levels. In consequence, the results must look "distorted" when compared with the expectation from Fig. 15; but, otherwise, a number of observations:

- polarization dependency: the $\mathrm{CD}$ values depend on the polarization,

- symmetry around the "carrier CD:" the CD values, measured at two perpendicular polarizations, are distributed symmetrically around the "carrier CD,"

- anti-symmetry for two targets,

- quasiparabolic $|\Delta \mathrm{CD}|$, and

- the location of the lowest $|\Delta \mathrm{CD}|$ at slightly positive focus level,

confirm all what is expected of the effective-CD. This gives us grounds to a claim that the effective-CD is indeed a real effect. It can be detected by a double, polarized scatterometric measurement, and it indeed reflects the presence of LER.

\section{Conclusions and Outlook}

In this paper, we have conclusively demonstrated that LER indeed has an impact on optical CD metrology. Moreover, 


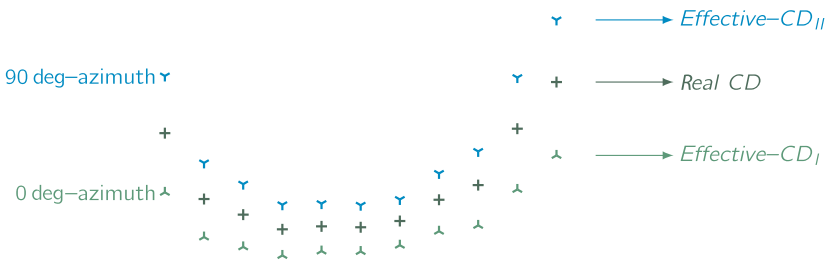

Fig. $17 \mathrm{~A}$ sketch schematically illustrating the main finding: in a scatterometric $C D$ measurement, the real $C D$ value is only to be found between two effective-CDs measured at two perpendicular polarizations. To create this sketch, we use data similar to data you can find in Fig. 16.

we have shown that this impact can be generalized by introducing the effective-CD, which existence we have proven experimentally. This means that the CD measurement should be revised according to the following sequence:

- perform a polarized scatterometric measurement twice, with polarizations perpendicular and parallel to the lines of the grating,

- the result will be two slightly different $C D$ values: $C D_{I}$ and $\mathrm{CD}_{\mathrm{II}}$. The fact that you measured two different $\mathrm{CD}$ values means that you have in fact measured effective$\mathrm{CD}_{\mathrm{I}}$ and effective- $C \mathrm{D}_{\mathrm{II}}$, which are certain virtual offsets from the real $\mathrm{CD}$ value,

- the real CD value ( = that is the CD that carries LER) is located between the two effective-CD values (see also Fig. 17):

$$
\text { effective- } \mathrm{CD}_{\mathrm{I}}<\text { real } \mathrm{CD}<\text { effective- } \mathrm{CD}_{\text {II }}
$$

Even more importantly, from the point of view of LER metrology, we have demonstrated that the difference between the two effective-CD values corresponds to LER:

$$
\mid \text { effective- } \mathrm{CD}_{\text {II }}-\text { effective- } \mathrm{CD}_{\mathrm{I}} \mid \rightarrow \text { LER }
$$

The latter statement means that LER measurement reduces to a double polarized $\mathrm{CD}$ measurement. If you use polarized light to measure the $\mathrm{CD}$ and you do it well, LER is already present in your results, waiting to be extracted!

The "reduced to a CD measurement" part of the statement above is the key take-home message that we want to convey with this paper. In Sec. 3, by presenting an overview of our simulation-based considerations, we try to convince you that regardless of the information channel that we look at (incidence angle $\alpha$, grating height $h$, wavelength $\lambda$, sidewall angle SWA, complexity of the roughness model, simulation engine, etc.), we see the same effective-CD coming back all the time. You need to keep in mind that all considerations in Sec. 3 are simulation-based ( = unaffected by any real-life measurement error sources) and of a very good convergence, which means that the $\mathrm{CD}$ offsets we observe can be neither measurement nor simulation artifacts. We are convinced that they are pure LER-induced effects.

The list of parameters (incidence angle $\alpha$, grating height $h$, etc.) seems to be complete from the scatterometric point of view, but for none of them could we break the apparently very rigid link between LER and the effective-CD, and decouple one from another. It may have some far-reaching implications. It is possible that, for any nonzero LER, the effective-CD is simply an intrinsic part of any optical CD measurement. It is possible that there exist no such information channels that allow for unbiased ( $=$ free from effectiveCD) CD measurement and LER measurement at the same time. It is possible that the only way to access the LER information is to perform two CD measurements (at two polarizations) and to utilize the effective-CD concept.

To summarize: when it comes to the future of LER metrology, we propose to consider the following chain of reasoning:

- LER metrology that uses the concept of the effective$\mathrm{CD}$ is not an independent metrology technique. On the contrary, it completely relies on the quality of CD metrology,

- consequently, LER metrology can only be as good as CD metrology,

- therefore, key future focus should be on further improving the CD metrology,

- because such further improvements in CD metrology can automatically ("for free") take advantage of the effective-CD.

In a concluding remark, we want to again emphasize the fact that polarization discrimination is essential to $\mathrm{CD}$ metrology. Polarized CD measurement using just one polarization is a bad idea. If you do so, most probably you do not measure the $\mathrm{CD}$. You just measure one of the effective-CDs.

\section{Disclaimer and Further Reading}

With this paper, we intended to present the basic fact that any routinely used scatterometric CD metrology scheme can, in principle, be conveniently employed for LER metrology. With this intention in mind, we focused on "general" not "detailed" aspects:

- instead of presenting any sensitivity analysis, we demonstrated that the effective-CD should be considered constant for any values of $\alpha, h$, and $\lambda$. Any potential "insensitivity" of any parameter in our approach is purely a problem of CD metrology, not LER metrology

- instead of focusing on discontinuities in Fig. 7, we focus on the general fact that the effective-CD seems to be constant everywhere else. As before, discontinuities are an issue with a CD measurement, not with the postulated effective-CD.

You will find a much more extensive treatment of the subject in the report published by the Institut für Technische Optik of Stuttgart University: "Line Edge Roughness in Optical Critical Dimension Metrology."11 There, among other things, we discuss the above-mentioned discontinuities, as well as what changes in the effective-CD when the correlation length of roughness or roughness exponent change. Moreover, we present the analysis of the importance of the optical parameters (refractive index) for a reliable effective-CD determination. We also discuss whether or not the effective-CD postulate still holds when Fourier scatterometry is applied. 


\section{Acknowledgments}

The research leading to these results has received funding from the European Community's Seventh Framework Programme under Grant Agreement No. 215723. We would like to thank ASML for providing the FEM, its measurements and help with data analysis.

\section{References}

1. C. Mack, "Line edge roughness, parts 1-3," Microlith. World 16(1-3) (2007).

2. B. C. Bergner, T. A. Germer, and T. J. Suleski, "Effect of line-width roughness on optical scatterometry measurements," Proc. SPIE $\mathbf{7 2 7 2}$, 72720U (2009).

3. T. Schuster, Simulation of Light Diffraction on Cross Gratings and its Application for Scatterometry, Vol. 64, Reports of Institute of Applied Optics (ITO) of Stuttgart University (2010).

4. D. Dixit et al., "Sensitivity analysis and line edge roughness determination of 28-nm pitch silicon fins using Mueller matrix spectroscopic ellipsometry-based optical critical dimension metrology," J. Micro/ Nanolithogr. MEMS MOEMS 14(3), 031208 (2015).

5. J. Bischoff and K. Hehl, "Scatterometry modeling for gratings with roughness and irregularities," Proc. SPIE 9778, 977804 (2016).

6. G. Gallatin, SPIE Short Course SC886: Line Edge Roughness (2010).

7. B. Bilski, K. Frenner, and W. Osten, "About the influence of line edge roughness on measured effective-CD," Opt. Express 19, 19967-19972 (2011).

8. M. Totzeck, "Numerical simulation of high-NA quantitative polarization microscopy and corresponding near-fields," Optik 112(9), 399-406 (2001).

9. M. Navière and E. Popov, Light Propagation in Periodic Media, Marcel Dekker (2003).

10. S. Rafler et al., "Investigation of methods to set up the normal vector field for the differential method," Proc. SPIE 6995, 69950Y (2008).
11. B. Bilski, Line Edge Roughness in Optical Critical Dimension Metrology, Vol. 81, Reports of Institute of Applied Optics (ITO) of Stuttgart University (2016).

12. Y. Zhang, M. Feng, and H.-Y. Liu, A Focus Exposure Matrix Model for Full Chip Lithography Manufacturability Check and Optical Proximity Correction (2006).

Bartosz Bilski holds a master's degree from Warsaw University of Technology and a doctoral degree from Stuttgart University. Both degrees were earned while working on optical subjects: interferometric and holographic tomography, and optical Critical Dimension metrology, respectively. In parallel to finalizing his doctoral thesis, Dr. Bilski worked on further developments of a parallel lens interferometer integrated into ASML's high-end DUV scanners. Currently, he is involved in imaging performance qualification of the future EUV systems.

Karsten Frenner received his doctorate in electrical engineering from Stuttgart University in 2009. Here, his research focused on discrete differential forms for electromagnetic simulations. Since 2007, he is leading the group "High Resolution Metrology and Simulation" at the Institute of Applied Optics, Stuttgart University.

Wolfgang Osten received his Diploma in physics from FriedrichSchiller-University Jena in 1979 and his PhD from Martin-LutherUniversity Halle-Wittenberg in 1983. From 1984 to 1991, he was at the Central Institute of Cybernetics and Information Processes in Berlin making investigations in digital image processing and computer vision. In 1991, he joined the Bremen Institute of Applied Beam Technology to establish the Department of Optical 3-D-Metrology. Since September 2002, he has been a full professor at the University of Stuttgart. 